\title{
Brevissime dalla Letteratura internazionale
}

\author{
Gianna d'Adamo
}

Nefrologa, Roma

\begin{abstract}
COST OF PERITONEAL DIALYSIS AND HAEMODIALYSIS ACROSS THE WORLD
Abstract. This report summarizes the scientific paper comparing the costs of hemodialysis (HD) and peritoneal dialysis (PD) in several countries, which was published on NDT in June 2013. After thorough literature review, the Authors found that PD is less expensive than HD in most developed countries. In developing countries the situation is rather variable and the costs of DP and HD are generally similar where economies of scale have been realized.
\end{abstract}

Key words: Peritoneal dialysis, Hemodialysis, Economic analysis, Dialysis cost

Conflict of interest: None.

Ricevuto: 7 Giugno 2013; Accettato: 9 Luglio 2013

\section{Costo della dialisi peritoneale e dell'emodialisi nel mondo}

\section{Cost of peritoneal dialysis and haemodialysis across the world}

Karopadi AN, Mason G, Rettore E and Ronco $C$. Nephrol Dial Transplant. 2013 Jun 4. [Epub ahead of print].

"Dialisi peritoneale: dialisi marginale". Su questo tema possiamo leggere le riflessioni aggiornate di molti esperti in uno spazio dedicato del sito di questo giornale a cui potrebbe seguire un numero speciale dedicato con una selezione, fortemente voluto dall'Editor Dr. Lombardi e dal Dr. Corciulo, Coordinatore del Gruppo di Studio di DP della Società Italiana di Nefrologia. A 12 anni dal primo Supplemento del 2001, che ha affrontato questo stesso tema, e in tempo di crisi economica, l'importanza del costo del trattamento sostitutivo è cresciuta. Avere le idee chiare su questo aspetto aiuterà a comprendere meglio le molte componenti che concorrono alla persistente marginalità della dialisi peritoneale. Nel recentissimo lavoro appena pubblicato su NDT sul costo dell'emodialisi e della dialisi peritoneale in diversi Paesi, gli Autori hanno condotto un'analisi approfondita della letteratura selezionando 78 articoli relativi a 46 Paesi. I diversi studi presentavano una grande variabilità quanto a periodo di effettuazione, obiettivi e scelta degli elementi e/o delle implicazioni economiche da includere nel calcolo del costo dei trattamenti.

Per quasi tutti i Paesi, gli Autori hanno deciso di considera- re solo i lavori pubblicati dal 2005 in poi; solo per 6 Paesi hanno dovuto considerare lavori meno recenti. Per 8 Paesi in via di sviluppo (Cina, Corea del Sud, Bangladesh, Nepal, Tailandia, Taiwan, Argentina, El Salvador) con un alto tasso di pazienti in DP e/o nuove politiche sanitarie, gli Autori hanno considerato anche i costi stimati da nefrologi locali. Per ogni Paese è stato calcolato il rapporto tra costo della HD e costo della DP, superando, così, anche problemi di valuta e/o cambio.

I dati raccolti riguardano 46 Paesi, di cui 26 in via di sviluppo, e il rapporto tra i costi dei trattamenti varia molto tra $\mathrm{i}$ diversi Paesi.

In 22 Paesi, 17 sviluppati e 5 in via di sviluppo, il costo della HD era da 1.25 a 2.35 volte maggiore rispetto a quello della DP; in 15 Paesi, 2 sviluppati (Germania e Grecia) e 13 in via di sviluppo, era da 0.9 a 1.25 volte maggiore rispetto a quello della DP. In altri 9 Paesi, di cui uno solo sviluppato (Giappone), il rapporto tra costo di HD e DP era compreso tra 0.90 e 0.22 .

Per quanto riguarda 1'Italia, gli Autori hanno esaminato 9 studi (1-9) e considerato pari a 1.81 il rapporto tra costo della HD e costo della DP, sulla base dello studio più recente relativo al solo costo base dei trattamenti, senza includere, quindi, i costi di complicanze, trasporti, perdita di produttività e via dicendo (9).

Nella maggior parte dei Paesi sviluppati, la DP è, quindi, un trattamento meno costoso rispetto alla HD. Invece, nei Paesi in via di sviluppo, la situazione è più varia e il costo della DP è simile a quello della HD, laddove sono state realizzate economie di scala abbattendo le tasse sull'importazione dei materiali o producendo le soluzioni per la dialisi peritoneale in loco. 


\section{Riassunto}

Breve riassunto del recentissimo lavoro sui costi dell'emodialisi e della dialisi peritoneale in diversi Paesi, pubblicato su NDT nel Giugno del 2013. Dopo un'approfondita revisione della letteratura, gli Autori concludono che, nella maggior parte dei Paesi sviluppati, la DP è un trattamento meno costoso rispetto alla HD, mentre, nei Paesi in via di sviluppo, la situazione è molto varia e il costo della DP è simile a quello della HD dove sono state realizzate economie di scala.
Parole chiave: Dialisi peritoneale, Emodialisi, Analisi economica, Costo della dialisi

Dichiarazione di conflitto di interessi: L'Autore dichiara di non avere conflitto di interesse.

Indirizzo degli Autori:

Dr.ssa Gianna d'Adamo

Nefrologa

Roma

giannadadamo@gmail.com

\section{Bibliografia}

1. Baroni A, Airoldi Savoini GP, Ragazzoni E, et al. Metodologie dei costi in dialisi in rapporto all'azienda salute italiana. [Cost methodologies in dialysis compared to the Italian National Health Service]. Minerva Urol Nefrol 1994; 40: 17-22.

2. Brunetti M, Pagano E, Garattini L. Il costo della dialisi peritoneale e dell'emodialisi nei centri ad assistenza limitata in Italia. [Cost of peritoneal dialysis and hemodialysis in limited care facilities in Italy]. ASI 1998; 9: 39-42.

3. De Negri A, Formica M, Cavagnino A. Valutazione dei costi dei trattamenti dialitici. [Evaluation of the cost of dialysis modalities]. In: Rapporto della commissione dialisi, parte C. G Ital Nefrol 1997; 14: 5-9.

4. La Greca G, Chiaramonte S, Feriani M, et al. Substitutive treatment in end-stage renal disease: hemodialysis versus peritone- al dialysis. Contrib Nephrol 1994; 109: 45-52.

5. Maiorca R, Sanfrini S, Cancarini GC, et al. Integration of peritoneal dialysis and transplantation programs. Perit Dial Int 1997; 2: S170-4.

6. Martina G, Boero R, Forneris G, et al. I trattamenti dialitici extraospedalieri. [Dialysis treatments outside the hospital]. Minerva Urol Nefrol 1994; 46: 1-5.

7. Jeantet A, Piccoli GB, Pacitti A, et al. [Costs of dialysis in hospitalized patients with acute or chronic renal failure, according to area of treatment]. G Ital Nefrol 2002; 19: 308-15.

8. Tediosi F, Bertolini G, Parazzini F, et al. Cost analysis of dialysis modalities in Italy. Health Serv Manage Res 2001; 14: 9-17.

9. Pontoriero G, Pozzoni P, Del Vecchio L, et al. International study of Health Care Organization and financing for renal replacement therapy in Italy: an evolving reality. Int J Health Care Finance Econ 2007; 7: 201-15. 